\title{
Infección profunda del cuello por Streptococcus grupo B en un paciente diabético. Caso clínico
}

Jorge Sapunar $Z^{1}$, Alvaro Cabello $\mathbf{V}^{2}$, Emilio G odoy $\mathbf{R}^{3}$.

Retropharyngeal phlegmon caused by a group B Streptococcus in a diabetic patient. Report of one case

We report a 47 year-old diabetic male, admitted due to metabolic decompensation, malaise, purulent pharyngeal discharge and a mass in the posterior cervical region. Blood glucose was $270 \mathrm{mg} / \mathrm{dl}$, a nasopharyngoscopy showed a pharyngeal phlegmon and CT scan confirmed the presence of a phlegmon in the retropharyngeal region. He was treated with sodium penicillin, cloxacillin and ceftriazone and the phlegmon was drained surgically. The culture of the purulent discharge gave growth to a Group B Streptococcus. The evolution was favorable and the patient completed seven days with intravenous antimicrobials and additional seven days with oral ampicillin/sulbactam (Rev Méd Chile 2008; 136: 351-5).

(Key words: Cellulitis; Pharynx; Streptococcus)

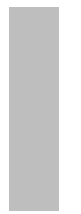

\begin{abstract}
Recibido el 13 de marzo, 2007. Aceptado el 9 de julio, 2007.
${ }^{1}$ Unidad de Endocrinología y Diabetes, Departamento de Medicina Interna, Facultad de Medicina de la Universidad de La Frontera y Clínica Alemana de Temuco. ${ }^{2}$ Cirugía de Cabeza y Cuello, Servicio de Cirugía, Hospital Regional de Temuco Hernán Henríquez Aravena y Clínica Alemana de Temuco. ${ }^{3}$ Otorrinolaringología, Clínica Alemana de Temuco.
\end{abstract}

$\mathrm{L}$ a diabetes mellitus (DM) se asocia con una mayor susceptibilidad a infecciones ${ }^{1}$. La condición de diabético se asocia con un discreto aumento en el riesgo de morir por neumonía y de presentar complicaciones como derrame pleu$\mathrm{ral}^{2,3}$. La mayor frecuencia de infección del tracto

Correspondencia a: Dr. Jorge Sapunar Z. Dinamarca 621 Of 601, Temuco. IX Región. Fono/Fax: 045/735778.

E mail: jsapunar@ufro.cl urinario entre mujeres diabéticas ha sido cuestionada desde la incorporación del concepto de bacteriuria asintomática $^{4,5}$. Sin embargo, el desarrollo de infecciones urinarias altas o complicaciones de éstas es mucho más frecuente entre los diabéticos $^{6}$. La DM es un factor de riesgo para desarrollar tuberculosis, septicemias por Staphylococcus aureus resistente a meticilina, Streptococcus del grupo B (agalactiae) y hongos del género Candida ${ }^{7-10}$. Existen infecciones poco frecuentes, pero muy graves, que se observan casi 
exclusivamente en pacientes diabéticos, como la otitis externa invasora, la mucormicosis rinocerebral y las infecciones enfisematosas ${ }^{11-13}$.

Entre las infecciones que afectan a los pacientes diabéticos destacan por su alta prevalencia y frecuentes complicaciones aquellas que afectan las partes blandas de las extremidades inferiores ${ }^{14}$. Se debe mencionar también la fasciitis necrotizante, que puede afectar diversos segmentos corporales y se caracteriza por su elevada mortalidad $^{15,16}$.

Se presenta un caso de infección en un paciente diabético causada por un agente microbiológico inhabitual, con presentación clínica atípica y evolución larvada.

\section{Caso Clínico}

Varón de 47 años con DM diagnosticada hace 9 años, en tratamiento actual con esquema insulínico intensivo (una dosis nocturna de insulina glargina e insulina lispro previo a las comidas). Entre sus antecedentes destacaban gran tabaquismo, dislipidemia mixta en tratamiento irregular con ciprofibrato $100 \mathrm{mg} /$ día y microalbuminuria de $50 \mathrm{mg} / 12 \mathrm{~h}$, sin hipertensión arterial en tratamiento con enalapril $5 \mathrm{mg} /$ día.

Consultó en un centro de salud por sensación febril no cuantificada, calofríos, odinofagia, náuseas y vómitos alimentarios de 2 días de evolución. Se constató deshidratación moderada, temperatura axilar de $37,8^{\circ} \mathrm{C}$ y glicemia capilar mayor de 400 $\mathrm{mg} / \mathrm{dl}$. Fue internado, recibiendo solución fisiológica e insulina cristalina en esquema de minidosis. Entre los exámenes de laboratorio obtenidos al ingreso destacaban glicemia $549 \mathrm{mg} / \mathrm{dl}$, creatinina $1,67 \mathrm{mg} / \mathrm{dl}, \mathrm{pH}$ 6,91, $\mathrm{BE}-27, \mathrm{HCO}_{3}$ 4,6 (en sangre arterial), cetonemia +++ , natremia $141,7 \mathrm{mEq} / \mathrm{L}$, kalemia $5,92 \mathrm{mEq} / \mathrm{L}$, cloremia $98 \mathrm{mEq} / \mathrm{L}$, amilasemia $48 \mathrm{U} / \mathrm{L}$, hematocrito 40,5\%, leucocitos 9.100 $\mathrm{mm}^{3}$, VHS $38 \mathrm{~mm} / \mathrm{h}$ y urocultivo negativo. Fue dado de alta con el diagnóstico de cetoacidosis diabética, se le indicó mantener el esquema insulínico intensificado y asistir a control ambulatorio. El paciente se trasladó a la ciudad de Temuco, consultando 10 días después del alta por sensación febril no cuantificada, sudoración nocturna, odinofagia y dolor al movilizar el cuello. Al examen físico destacaba un gran aumento de volumen de la región posterior del cuello, sensible, sin signos inflamatorios ni crepitación, fetor pútrido y faringe congestiva con escaso exudado purulento. Se indicó hospitalización con el diagnóstico de DM descompensada, faringo-amigdalitis pultácea y masa cervical en estudio. Entre los exámenes destacaban glicemia $270 \mathrm{mg} / \mathrm{dl}$, creatinina $0,8 \mathrm{mg} /$ $\mathrm{dl}$, hematocrito $35,7 \%$, leucocitos 18.800 por $\mathrm{mm}^{3}$, VHS $91 \mathrm{~mm} /$ hora, PCR 309 mg/L. Se inició hidratación con solución fisiológica e infusión endovenosa continua de insulina, fue evaluado por otorrinolaringólogo con nasofaringoscopia, pesquisándose flegmón faríngeo. Se inició terapia con penicilina sódica EV 20 millones U/día, cloxacilina 12 g EV/ día y ceftriaxona $2 \mathrm{~g} \mathrm{EV/día.} \mathrm{El} \mathrm{aumento} \mathrm{de}$ volumen cervical fue estudiado con ecotomografía cervical, que mostró una colección en el compartimiento posterior del cuello (Figura 1) y tomografía axial computarizada multicorte de cuello en que se apreciaba aumento de volumen del espacio retrofaríngeo y de los compartimientos posteriores del cuello de aspecto flegmonoso (Figura 2).

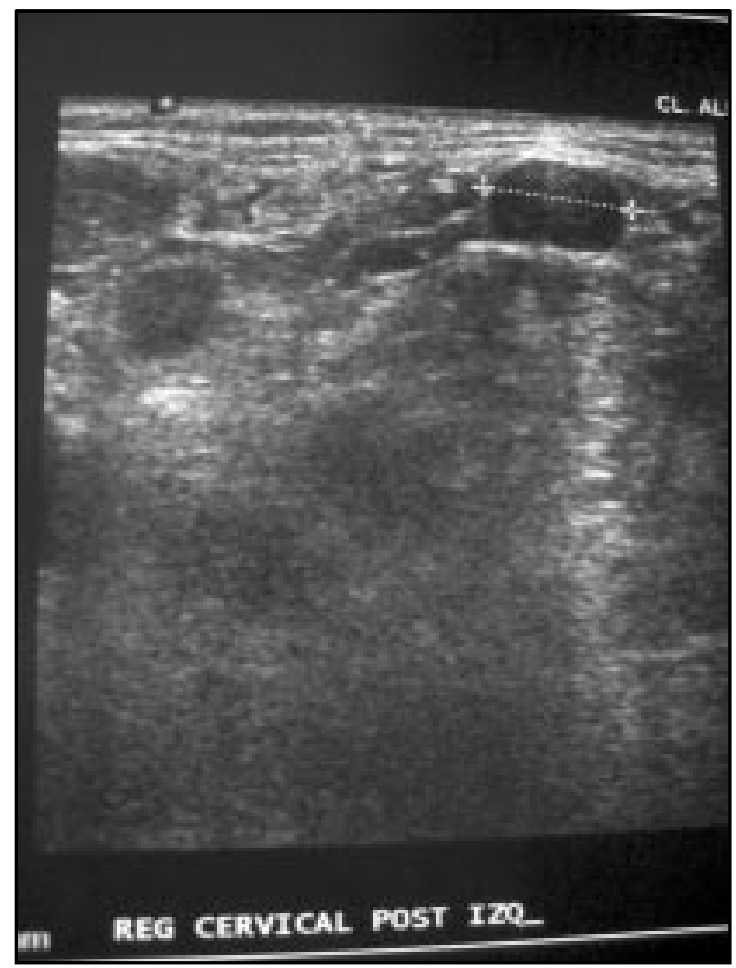

Figura 1. Ecotomografía de los planos faciales posteriores del cuello, donde se aprecia colección líquida. 


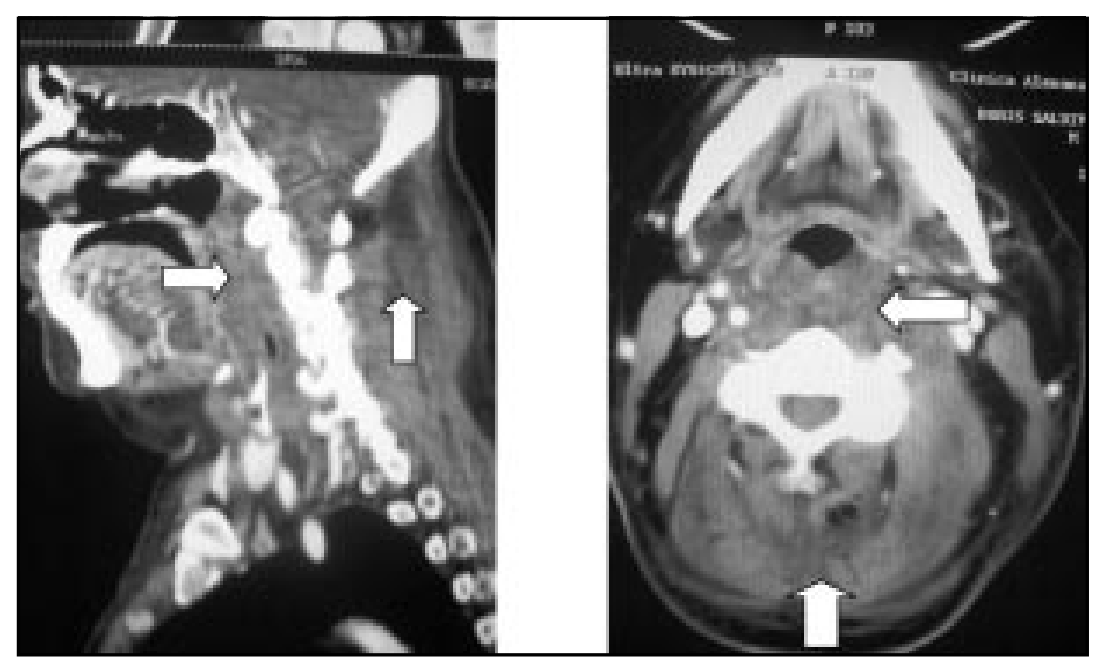

Figura 2. Tomografía axial computarizada multicorte cervical donde se aprecia compromiso del espacio retrofaríngeo y planos faciales posteriores.

Evaluado en conjunto con otorrinolaringólogo y cirujano de cabeza y cuello, se decidió practicar drenaje quirúrgico de la colección. Al segundo día de hospitalización se realizaron 2 incisiones en la región posterior del cuello, drenando líquido purulento. No se encontró evidencias de necrosis o gas. Se obtuvo cultivo de la secreción, aislándose Streptococcus grupo B, los hemocultivos fueron negativos. Luego de una semana con antibióticos EV, los drenajes Penrose dejaron de entregar secreción y fueron retirados. La exploración de las incisiones fue satisfactoria. Se dio de alta con antibiótico oral hasta completar otra semana (Ampicilina/Sulbactam).

\section{DISCUSIÓN}

Las infecciones profundas del cuello incluyen los flegmones y abscesos de los planos faciales y espacios virtuales como el retrofaríngeo. Debido al desarrollo de complicaciones a menudo letales, como obstrucción de la vía aérea, mediastinitis, trombosis venosa, embolia séptica, ruptura de arteria, pericarditis y septicemia, estas infecciones requieren tratamiento enérgico, con asociaciones de antibióticos y drenaje quirúrgico ${ }^{17}$. La DM es la enfermedad que más frecuentemente se asocia con el desarrollo de infecciones profundas del cuello ${ }^{18}$. Huang y cols, en un estudio retrospectivo de 185 pacientes con infecciones profundas del cuello, encontraron que los pacientes diabéticos tenían hospitalizaciones más prolongadas, mayor frecuencia de complicaciones y que el espacio más frecuentemente comprometido era el parafaríngeo. El origen de la infección sólo se pudo determinar en $43,7 \%$ de los casos y fue mayoritariamente oral. Fue posible identificar el microorganismo en 60\% de los pacientes, siendo los gérmenes más frecuentemente aislados Streptococcus viridans y Peptostreptococcus entre los no diabéticos y Klebsiella pneumoniae en diabéticos ${ }^{19}$. Chang y cols, en una revisión de 100 casos de infecciones de los espacios fasciales del cuello ${ }^{20}$, encontraron que el microorganismo más frecuentemente aislado era Klebsiella pneumoniae (48\%) y que esta frecuencia aumentaba significativamente al considerar sólo a los pacientes diabéticos (81,3\%). Nuestro paciente desarrolló infección del espacio retrofaríngeo y planos fasciales posteriores cuyo compromiso, según Huang y cols, es más frecuente entre los diabéticos. En nuestro caso, el origen de la infección profunda fue la vía aérea superior, más frecuente en no diabéticos en el estudio de Huang y el germen causal fue un Streptococcus grupo B, germen no pesquisado en esa casuística ${ }^{19}$. 
El Streptococcus grupo B está involucrado en infecciones graves en recién nacidos, mujeres en período perigestacional y ancianos con enfermedades crónicas. El principal reservorio para esta bacteria es el tracto gastrointestinal inferior, la colonización orofaríngea sólo es frecuente en homosexuales ${ }^{21}$. Se ha observado una tendencia al aumento de las infecciones por este microorganismo en adultos no incluidos en los subgrupos antes mencionados, que comprometen principalmente piel y partes blandas, articulaciones y huesos, menos frecuentemente pulmón y tracto urinario $^{22,23}$. Huang y cols en un análisis retrospectivo de una serie de 94 pacientes adultos no gestantes con infección por Streptococcus grupo $\mathrm{B}$, encontraron que $42,6 \%$ padecían diabetes mellitus, 31,9\% tenían una infección de partes blandas. La mortalidad fue 20,2\%, Klebsiella pneumoniae y Staphylococcus aureus fueron los microorganismos asociados más frecuentes?.

En una serie de casos clínicos de espondilitis, 2 de 20 pacientes tenían compromiso de vértebras cervicales, sin embargo no se menciona compromiso de planos fasciales o espacios virtuales. El tratamiento antibiótico empleado en un caso fue ceftriaxona y en el otro penicilina ${ }^{24}$.

Junto a Klebsiella pneumoniae, Streptococcus viridans, Peptostreptococcus y Streptococcus grupo $B$ se deben considerar otros microorganismos en las infecciones profundas del cuello. Chi y cols reportaron un absceso retrofaríngeo con extensión epidural y osteomielitis cervical por Aspergillus flavus ${ }^{25}$. Custal-Teixidor y cols, en una serie de 15 casos de infección por Actinomyces sp, reportaron un caso de absceso parafaríngeo. En este último grupo de agentes (Actinomyces israeli, naeslundi, odontolyticus, viscosus, meyeri y Propionibacteriumpropionicum) la infección se presenta clínicamente como tumor cervical cuyo foco de origen es oral26.

\section{REFERENCIAS}

1. Joshi N, Caputo MG, Weitekamp Mr, Karchmer AW. Infections in patients with diabetes mellitus. N Engl J Med 1999; 341: 1906-12.

2. Fine MJ, Smith MA, Carson CA. Prognosis and outcomes of patients with community-adquired pneumonia: a meta-analysis. JAMA 1996; 275: 134-41.
Entre las infecciones profundas del cuello destaca por su letalidad la fasciitis necrotizante. Si consideramos algunos de los criterios que definen la angina de Ludwig como la ausencia de pus, necrosis tisular con formación de gas, compromiso secundario de músculos y piel, nuestro caso no correspondía a fasciitis necrotizante ${ }^{27}$. Mathieu y cols reportaron una serie clínica de 45 casos de fasciitis necrotizante cervical ${ }^{28}$ cuyos hallazgos más relevantes fueron que ésta sólo representó 9,5\% de las infecciones cervicales referidas para hospitalización, que sólo $13 \%$ de los pacientes de la serie tenían DM y 58\% eran personas previamente sanas. En $78 \%$ de los casos, el origen de la infección fue oral y en $16 \%$ faríngeo. Hubo diseminación hacia la parte baja del cuello en 56\% y a mediastino en $40 \%$. En esta misma serie, $78 \%$ de los pacientes tuvieron cultivo positivo de muestras de tejido, encontrándose sólo un microorganismo anaerobio en 16\% y flora mixta anaerobia en 56\%. Los microorganismos anaerobios más frecuentemente aislados fueron Prevotella melaninogenica, Fusobacterium nucleatum y Peptostreptococcus. Bacterias del género Clostridium sólo se encontraron en 7\% y correspondían a infecciones de herida operatoria. Todos los pacientes fueron sometidos a cirugía y se utilizó metronidazol, penicilina, piperacilina y amikacina como antibióticos, en algunos casos oxígeno hiperbárico, a pesar de lo cual la mortalidad fue $22 \%$.

En conclusión, las infecciones profundas del cuello son más frecuentes en diabéticos, el foco de origen suele ser oral o faríngeo, el espacio cervical habitualmente afectado es el parafaríngeo, el microorganismo más frecuentemente aislado es Klebsiella pneumoniae aunque deben ser considerados Streptococcus viridans, Peptostreptococcus, Aspergillus flavus, Actinomyces sp y Streptococcus grupo B. La fasciitis necrotizante cervical es poco frecuente y la mayor parte de los afectados no tienen antecedentes mórbidos.

3. Falguera M, Pifarre R, Martin A, Sheikh A, Moreno A. Etiology and outcome of community-acquired pneumonia in patients with diabetes mellitus. Chest 2005; 128: 3233-9.

4. Andriole VT. Asintomatic bacteriuria in patients with diabetes enemy or innocent visitor. N Engl J Med 2002; 347: 1617-8.

5. Bonadio M, Costarelli S, Morelli G, Tartaglia T. 
The influence of diabetes mellitus on the spectrum of uropathogens and antimicrobial resistence in elderly adult patients with urinary tract infection. BMC. Infectious Diseases 2006; 6: 54-61.

6. Edelstein H, McCabe RE. Perinephric abscess: modern diagnosis and treatment in 47 cases. Medicine (Baltimore) 1988; 67: 118-31.

7. Ponce de León A, García-García M, García-Sancho MC, Gómez-Pérez FJ, Valdespino-Gómez JL, OlaizFERNÁNDEZ G ET AL. Tuberculosis and diabetes in southern México. Diabetes Care 2004; 27: 1584-90.

8. Chi C, Wong W, Fung C, Yu K, Liu C. Epidemiology of community-acquired Staphylococcus aureus bacteremia. J Microbiol Immunol Infect 2004; 37: 16-23.

9. Huang P, Lee M, Yang C, Leu H. Group B streptococcal bacteremia in non-pregnant adults. J Microbiol Immunol Infect 2006; 39: 237-41.

10. Cheng Y, Lin L, Young T, Liu C, Chen C, Tsay R. Risk factors for candidemia-related mortality at a medical centre in central Taiwan. J Microbiol Immunol Infect 2006; 39: 155-61.

11. Morales PP, Licea Me, Perera JJ. Otitis maligna del diabético: Nuestra experiencia y revisión de la literatura. Rev Cubana Endocrinol 2002; 13: 7-16.

12. Tierney MR, BaKer AS. Infections of the head and neck in diabetes mellitus. Infect Dis Clin North Am 1995; 9: 195-216.

13. Mentzer RM Jr, Golden GT, Chandler JG, Horsley JS III. A comparative appraisal of emphysematous cholecystitis. Am J Surg 1975; 129: 10-5.

14. Caputo GM, Cavanagh PR, Ulbrecht JS, Gibbons GW, Karchmer AW. Assessment and management of foot disease in patients with diabetes. N Engl J Med 1994; 331: 854-60.

15. Sentochnik DE. Deep soft-tissue infections in diabetic patients. Infect Dis Clin North Am 1995; 9: 53-64.

16. Lauks SS II. Fournier's gangrene. Surg Clin North Am 1994; 74: 1339-52.

17. Wills PI, Vernon RP Jr. Complications of space infections of the head and neck. Laryngoscope 1981; 91: 1129-36.

18. Parhiscar A, Har-El G. Deep neck abscess: a retrospective review of 210 cases. Ann Otol Rhinol Laryngol 2001; 110: 1051-4.

19. Huang T, Tseng F, Liu T, Hsu C, Chen Y. Deep neck infection in diabetic patients: Comparison of clinical picture and outcomes with nondiabetic patients. Otolaryngol Head Neck Surg 2005; 132: 943-7.

20. Chang CM, Lu FH, Guo HR, Ko WC. Klebsiella pneumoniae fascial space infections of the head and neck in Taiwan: emphasis on diabetic patients and repetitive infections. J Infect 2005; 50: 34-40.

21. Edwards MS, BaKer CJ. Streptococcus Agalactiae. En Mandell GL, Bennett JE, Dolin R, eds. Principles and Practice of Infectious Diseases. Philadelphia, Churchill Livingstone Company, 2000; 2615-28.

22. Farley MM. Group B streptococcal disease in nonpregnant adults. Clin Infect Dis 2001; 33: 55661.

23. Tyrrell GJ, Senzilet lD, Spika JS, Kertesz DA, Alagaratnam M, Lovgren M et al. Invasive disease due to group B streptococcal infection in adults: results from a Canadian, population-based, active laboratory surveillance study 1996. Sentinel Health Unit Surveillance System Site Coordinators. J Infect Dis 2000; 182: 168-73.

24. Solís-García J, Martínez-Alfaro E, Abad L, Solera J. Vertebral osteomyelitis caused by Streptococcus Agalactiae. J Infect 2000: 41: 84-90.

25. Cні C, Fung C, Liu C. Aspergillus flavus epidural abscess and osteomielitis in a diabetic patient. J Microbiol Immunol Infect 2003; 36: 145-8.

26. Custal-Teixidor M, Trull-Gimbernat JM, GarijoLópez G, Valldosera-Rosello M. Fine-needle aspiration cytology in the diagnosis of cervicofacial actinomycosis: report of 15 cases. Med Oral Patol Oral Cir Bucal 2004; 9: 1-6.

27. Moreland LW, Corey J, McKenzie R. Ludwig's angina: report of a case and review of the literature. Arch Intern Med 1988; 148: 461-6.

28. Mathieu D, Neviere R, Teillon C, Chagnon Jl, Lebleu N, Wattel F. Cervical necrotizing fasciitis: Clinical manifestations and management. Clin Infect Dis 1995; 21: 51-6. 